\section{Dicing with lung cancer}

Non-coding RNAs, such as microRNAs (miRNAs), have made a big splash in cancer research in recent years, prompting the examination of how these RNAs are regulated. Several recent publications, including one in Science, indicate that mutations in the machinery that processes non-coding RNAs are also selected for during tumorigenesis.

Pleuropulmonary blastoma is a rare form of lung cancer that arises in young children and is associated with an increased familial risk of developing cancer. Children with this disease are born with cysts in the lungs that contain a layer of epithelial cells over a dense layer of mesenchymal cells. It is the mesenchymal cells that form the blastomas. D. Ashley Hill and colleagues mapped a disease locus to chromosome 14q using four families with a history of pleuropulmonary blastoma. Of the 72 genes present in this area the authors pursued DICER 1 on the basis of the fact that loss of Dicer 1 in mouse lungs results in cysts and deregulated growth of mesenchymal cells. DICER1 encodes an RNase III endonuclease that is involved in the maturation of
miRNAs and small interfering RNAs (siRNAs). Further analyses in an additional seven families identified heterozygous germline mutations in DICER 1, which in most of the families resulted in a truncated protein that is likely to be non-functional. There were normal heterozygous individuals in all the families studied, implying that the loss of one DICER1 allele is not sufficient to induce pleuropulmonary blastoma. Intriguingly, immunohistochemistry studies indicated that the expression

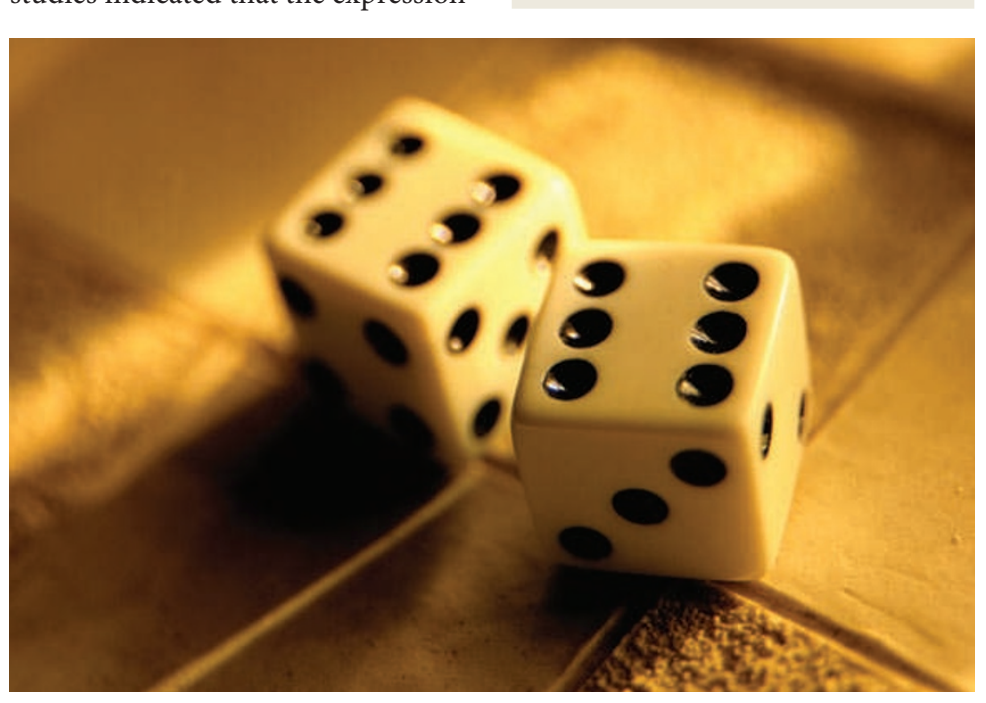

of DICER1 is lost in the epithelial cells in six of seven familial pleuropulmonary blastomas, but is retained in the mesenchymal tumour cells.

On the basis of these data, the authors suggest that pleuropulmonary blastoma arises as a result of alterations in miRNA or siRNA regulation in the epithelial cells. Such changes might result in altered growth factor or cytokine production, which could then induce transformation of the underlying mesenchymal cells.

Nicola McCarthy

ORIGINAL RESEARCH PAPER Hill, D. A. et al. DICER1 mutation in familial pleuropulmonary blastoma. Science 25 Jun 2009 (doi:10.1126/ science.1174334) 